\title{
Promovendo a saúde da pessoa amputada: uma açáo educativa chamada conversa no leito
}

\author{
Ruy Luiz Lorenzetti Branco, Kadine Priscila Bender dos Santos, \\ Soraia Cristina Tonon da Luz
}

Universidade do Estado de Santa Catarina - UDESC, Florianópolis, SC, Brasil.

\begin{abstract}
Resumo: Objetivo: O objetivo foi relatar a ação educativa intitulada "Conversa no Leito" a partir de cinco temas geradores: Reabilitação; Empoderamento; Amputação; Incertezas e Orientação. Essa pesquisa foi realizada no projeto de extensão Reabilitação Multidisciplinar em Amputados da Universidade do Estado de Santa Catarina. As ações do projeto em nível hospitalar incorporam: coleta das notificações; conversa no leito; entrega de um kit com folders informativos e faixa elástica para o enfaixamento do coto; agendamento para a avaliação fisioterapêutica no projeto de extensão pós-alta. Método: Este estudo de caso qualitativo foi realizado com uma pessoa recém-amputada de membro inferior num hospital público do município de São José/Santa Catarina. Resultados: Esta ação mostrou-se eficiente principalmente no empoderamento do paciente para o início precoce da reabilitação. Conclusão: Salienta-se atenção para capacitação da equipe multidisciplinar hospitalar envolvida quanto a orientações sobre protetização, retorno ao trabalho e esclarecimento de dúvidas gerais com atenção aos anseios do paciente e suporte familiar.
\end{abstract}

Palavras-chave: Amputação, Cuidados Pós-operatórios, Serviço Hospitalar de Fisioterapia, Educação em Saúde.

\section{Promoting the health of amputees: an educational action called talking with the bedridden patient}

\begin{abstract}
Objective: The aim of the present study was to report the educational activity called 'Talking with the Bedridden Patient' based on five themes: Rehabilitation; Empowerment; Amputation; Uncertainties and Guidance. This research was conducted as part of the outreach project Multidisciplinary Rehabilitation of Amputees of the State University of Santa Catarina. The actions of the project at hospital level included: collection of notifications; conversation with bedridden patients; delivery of a kit with informative brochures and an elastic band for the bandaging of the stump; schedule for physical therapy assessment after hospital discharge in the outreach project. Method: This qualitative case study was performed with a person who had recently undergone a lower limb amputation in a public hospital in the city of São José/Santa Catarina. Results: This project proved to be efficient mainly to empower the patient for early onset of rehabilitation. Conclusion: We call attention to the importance of training hospital multidisciplinary teams involved in providing guidelines on fitting, return to work and clarification of general doubts taking into account the patient's wishes and family support.
\end{abstract}

Keywords: Amputation, Postoperative Care, Physical Therapy Department, Health Education.

\footnotetext{
Autor para correspondência: Ruy Luiz Lorenzetti Branco, Laboratório de Biomecânica, Centro de Ciências da Saúde e do Esporte, Universidade do Estado de Santa Catarina, Rua Pascoal Simone, 385, Coqueiros, CEP 88080-350, Florianópolis, SC, Brasil, e-mail: ruy.1.branco@hotmail.com Recebido em Jul. 13, 2016; $1^{\text {a }}$ Revisão em Jan. 24, 2017; Aceito em Fev. 21, 2017.
} 


\section{Introdução}

Atualmente a amputação de membros inferiores é um grande problema de saúde pública, sendo uma condição de saúde crônica comum e importante causa de incapacidade em longo prazo às pessoas acometidas (CHINI; BOEMER, 2007).

Estimativas da prevalência de amputação de membros inferiores foram contabilizadas nos últimos cinco anos no Brasil, totalizando 102.056 cirurgias de amputação de membros inferiores, o que corresponde a 94\% dos procedimentos cirúrgicos realizados pelo Sistema Único de Saúde (SUS) (BRASIL, 2015). As principais etiologias destacadas foram: patologias oncológicas e congênitas, amputaçóes traumáticas e doenças cardiovasculares, com destaque para o diabetes, que ocupa a quarta posição nacional no ranking das amputaçôes de membros. Em nível nacional, os gastos relativos aos procedimentos de amputação somente no ano de 2014 chegaram a 18,2 milhóes atribuíveis ao diabetes (BRASIL, 2005).

As amputaçôes de membros inferiores impactam no aspecto biopsicossocial das pessoas, pois se caracterizam por uma perda física que restringe de forma importante a mobilidade e a independência funcional das pessoas, diminuindo também sua capacidade laborativa, qualidade de vida e socialização (CARVALHO et al., 2005). Assim, sugere-se que a reabilitação da pessoa amputada se inicie de preferência no período pré-cirúrgico (amputaçóes programadas) e pós-cirúrgico imediato, através de uma equipe multiprofissional que atue com um enfoque na atenção integral.

O procedimento de amputação é um processo que altera a vida da pessoa acometida, sendo a educação em saúde no ambiente hospitalar uma ação que pode ajudar no processo de reabilitaçáo (UNITED..., 2007). O ambiente hospitalar tem a capacidade de promover a saúde através de ações da equipe envolvida no cuidado e empoderamento da pessoa após a alta hospitalar, para que esta inicie precocemente o processo de reabilitação no ambiente ambulatorial (SILVA et al., 2011).

Tendo em vista que as pessoas que sofreram uma amputação de membro inferior após a alta hospitalar necessitam iniciar a reabilitação a fim de prevenir incapacidades que podem se tornar irreversíveis, e ainda preocupados com a promoçáo da saúde de forma a atender suas reais necessidades, este estudo buscou responder à seguinte questão de pesquisa: Como uma ação educativa individualizada no ambiente hospitalar, chamada Conversa no Leito pode contribuir para a promoção da saúde da pessoa amputada?
Na busca de respondermos a este questionamento, realizamos uma investigação qualitativa com pessoas amputadas no leito hospitalar, com o objetivo de relatar a ação educativa intitulada Conversa no Leito a partir de cinco temas geradores: Reabilitação; Empoderamento; Amputação; Incertezas e Orientação.

\section{Diretrizes de Atenção à Saúde da Pessoa Amputada}

Em uma pesquisa por materiais contendo orientaçôes para a saúde da pessoa amputada, fez-se uma busca em base de dados Medline, pubMed e Scielo, utilizando os seguintes idiomas: inglês, espanhol e português, tendo como palavras-chave em inglês: physiotherapy, amputation, health education, health promotion e post operative; em espanhol: fisioterapia, amputación, educación para la salud, promoción de la salud e postoperatorio; e em português: fisioterapia, educação em saúde, promoção da saúde e pós-operatório, tendo por delimitação de ano de 2006 a 2016, e como critério estudos clínicos randomizados, estudos de caso, revisôes sistemáticas, livros e guidelines. $\mathrm{O}$ que melhor se encontrou foram diretrizes e guidelines para a saúde da pessoa amputada, primeiramente diretrizes nacionais e posteriormente diretrizes internacionais. Encontraram-se uma diretriz e três guidelines, uma diretriz brasileira, três guidelines estrangeiros e uma revisão sistemática. A diretriz brasileira intitulada Diretrizes de Atenção à Pessoa Amputada, lançada em 2012 e publicada em 2013 pelo Ministério da Saúde. tem como objetivo orientar multidisciplinarmente as equipes para o cuidado à saúde da pessoa amputada nos diferentes pontos de atenção à Rede de Cuidados à Pessoa com Deficiência (BRASIL, 2012b). A Diretriz brasileira apresenta em sua estrutura o conceito de amputação, as etiologias das amputaçóes, estando dividida em três fases para melhor guiar os profissionais de saúde: Fase Pré-cirúrgica e cirúrgica, que consiste no preparo da pessoa ao processo cirúrgico, escolha do nível de amputação, nível de amputação de membros superiores e inferiores, avaliação dos aspectos cognitivos, preparação do paciente para o momento da notícia. A Fase Pós-cirúrgica imediata orienta os profissionais sobre os cuidados da pessoa no ambiente hospitalar logo após a amputação, transferências, deslocamentos, treino de marcha, orientação sobre o correto posicionamento a fim de evitar deformidades, controle do edema e modelagem do coto, exercícios de fortalecimento muscular e dessensibilização do coto. A terceira fase, chamada 
de Pré-protética, consiste na avaliação geral do coto de amputação, forma, presença de cicatrizes, infecção, distúrbios de sensibilidade, neuroma e espículas ósseas e a avaliação específica que avalia as medidas de comprimento e perimetria do coto. A Fase Protética orienta o paciente ao uso correto da prótese, sobre as transferências de peso e locomoção. Destacamos que esta Diretriz Nacional nos traz informaçóes sobre a importância do paciente em procurar a Atenção Básica para auxiliar seu processo de reabilitação (BRASIL, 2012b).

Em 2006 foi lançado em Londres o Clinical Guidelines for the pre and Post Operative Physiotherapy Management of Adults with Lower Limb Amputation (BROOMHEAD et al., 2006). Diferentemente da diretriz brasileira, que tem um enfoque multidisciplinar, este guideline objetiva informar aos fisioterapeutas o manejo correto de adultos com amputaçôes de membros inferiores no pré e pós-operatório, informar também a eficácia do tratamento baseando-se em evidências científicas.

O guideline está dividido em seis sessóes: o papel do fisioterapeuta dentro da equipe multidisciplinar, conhecimento, avaliação, informaçóes à pessoa e ao cuidador e manejo no pré e pós-operatório.

O departamento de veteranos de guerra, junto com o departamento de defesa dos Estados Unidos da América, lançou em 2008 o Clinical Practice Guideline for Rheabilitation of Lower Limb Amputation (UNITED..., 2007), um guideline que traz doze itens em sua composição: definiçáo das fases de cuidados no processo de reabilitação, incluindo os passos de cada fase, o reconhecimento da importância da avaliação da pessoa pela equipe multidisciplinar antes e após o processo cirúrgico, a importância da tomada de decisão sobre o nível de amputaçáo para cada pessoa, identifica os elementos-chave da reabilitação e treinamento com prótese, entre outros.

Em 2015, na Holanda, foi lançada uma revisão sistemática intitulada Dutch evidence-based guidelines for amputation and prosthetics of the lower extremity: Amputation surgery and postoperative management part 1 and part 2 (GEERTZEN et al., 2015a, b). Este artigo está dividido em Parte 1 e Parte 2. Na Parte 1 são abordados os critérios de indicação para amputação, técnicas cirúrgicas, informaçôes a pessoa, manejo no pós-operatório, controle da dor e complicaçóes. Na Parte 2, o foco direciona-se ao processo de reabilitação, aspectos psicossociais, fatores para a reabilitação e metas de treinamento, volta ao trabalho e fornecimento de próteses e seus componentes.

\section{Método}

Esta pesquisa faz parte de uma das ações do projeto de extensão Reabilitação Multidisciplinar em Amputados, pertencente ao programa Reabilitar e Integrar do Centro de Ciências da Saúde e do Esporte - CEFID da Universidade do Estado de Santa Catarina - UDESC. O projeto de extensão Reabilitação Multidisciplinar em Amputados tem suas atividades realizadas desde 2012. Fazem parte da equipe: docentes, mestrandos do Programa de Pós-graduação em Fisioterapia e discentes dos cursos de Fisioterapia e Educação Física.

Trata-se de um estudo da macropesquisa intitulada: Promoção da Saúde da Pessoa Amputada no Ambiente Hospitalar, que está sendo realizada desde 2013 com o apoio financeiro do edital chamada Universal 484010/2013 do CNPq, e do edital chamada 07/2013 Pesquisa para o SUS (PPSUS) FAPESC. O projeto foi baseado na Diretriz de Atenção à Pessoa Amputada (BRASIL, 2012b) e busca promover açôes intersetoriais para o acolhimento de pessoas que sofreram amputaçáo a partir do ambiente hospitalar.

As etapas deste macroprojeto se dividem em: notificação hospitalar, conversa no leito, avaliação fisioterapêutica, encaminhamento para fisioterapia ambulatorial no projeto de extensão e escola de marcha.

A confecção da ficha de notificação hospitalar foi realizada com o intuito de buscar auxílio na Rede de Atenção à Saúde da Pessoa Amputada, facilitando rapidamente o acesso à saúde e início do processo de reabilitação. A dinâmica engloba a visita do acadêmico do curso de Fisioterapia da UDESC, a fim de receber essas notificaçóes (dispostas em pastas em setores previamente instalados e anteriormente pactuado com os profissionais), a partir do preenchimento, pela equipe multidisciplinar do hospital, da ficha de notificação hospitalar.

O estudo relatado neste artigo caracteriza-se como um estudo de caso intrínseco, em que há um interesse sobre um caso em particular (STAKE, 2005) com uma abordagem qualitativa. A pesquisa foi realizada devido à chegada tardia das pessoas amputadas ao projeto de extensão, dificultando a sua reabilitação e futuro processo de protetização. O estudo foi feito em um hospital localizado no município de São José, no Estado de Santa Catarina, após a aprovação de dois comitês de ética: UDESC no 33009514.9.3001.0112 e do Hospital no 33009514.9.3001.0112, e está de acordo com a resolução CNS 466/12. 
Participou desta pesquisa um adulto de 24 anos, que passou por cirurgia de amputação transfemural decorrente de um acidente automobilístico e esteve hospitalizado durante seis dias no setor de ortopedia do referido hospital.

Esta ação educativa individualizada iniciou-se como uma das ações do projeto de extensão em virtude da demanda por uma ação educativa neste nível de complexidade, visando à promoção da saúde a partir do ambiente hospitalar. Constatamos que a maioria das pessoas chegava ao nosso projeto com o discurso da necessidade de uma ação orientadora no ambiente hospitalar para a prevençáo de sequelas músculo-esqueléticas muitas vezes irreversíveis, além de encaminhamentos para o início da reabilitação precoce. Delimitado o método, fez-se a calibração dos instrumentos de estudo, tais como o roteiro de conversa informal, gravador de áudio, diário de campo, a arte do kit a ser entregue ao paciente, composto por uma pequena bolsa contendo uma cartilha de orientaçáo e uma faixa para enfaixamento do coto e seleção de informaçóes pertinentes ao material direcionado para a promoção da saúde no ambiente hospitalar.

O diário de campo constituiu-se em um instrumento utilizado durante a conversa no leito onde são escritos impressóes pessoais, resultados de conversas informais, observaçóes de comportamento contraditório com as falas, expressôes dos interlocutores quanto a vários pontos investigados, entre outros (SOUZA, 2013). O reconhecimento do campo de estudo, a apresentação à equipe profissional, é de grande valia, pois permite o conhecimento prévio do ambiente e a conversa no leito a partir do conhecimento prévio sobre o entrevistado, respeitando as relaçóes interpessoais, hierarquia e burocracia (SZYMANSKI; ALMEIDA; PRANDINI, 2010).
A conversa no leito foi elaborada a partir de cinco temas geradores, ou seja, conteúdos que expressem as questóes norteadoras do roteiro de conversa entre pesquisador e pesquisado (GASKELL; BAUER, 2002). Os temas geradores são os seguintes: Reabilitação; Empoderamento; Amputação; Incertezas e Orientação, e o roteiro de conversa (Tabela 1 ).

\subsection{Procedimentos e coletas de dados}

No dia 19 de maio de 2015, às $17 \mathrm{~h} 00 \mathrm{~min}$, foi realizada a leitura do prontuário da pessoa disposto no posto de enfermagem no setor de ortopedia do hospital levantaram-se as seguintes informaçóes: nome, idade, endereço, história da doença atual, data da amputação, medicamentos utilizados e evolução do seu estado geral. Tais dados foram registrados no diário de campo do pesquisador. Aproveitou-se esse momento para perguntar à equipe sobre as condiçôes clínicas da pessoa, e foi informado que o paciente estava em bom estado geral.

A ação educativa chamada Conversa no Leito iniciou-se após apresentação do pesquisador ao pesquisado, leitura do Termo de Consentimento Livre e Esclarecido (TCLE), o objetivo desta pesquisa. foram também apresentados o projeto de extensão, o objetivo do projeto de extensão, atividades que sáo realizadas no projeto e a equipe que o compóe. A ação educativa dividiu-se em três momentos: o momento da apresentação da proposta, entrega de um kit e a conversa no leito.

O momento da entrega do kit teve início com a entrega de um kit educativo à pessoa. O kit educativo (Figura 1), acondicionado em uma bolsa, contém uma cartilha de orientaçóes intitulada Atenção à Saúde da Pessoa Amputada, um folder informativo sobre o projeto de extensão Reabilitação Multidisciplinar em Amputados e uma faixa elástica para enfaixamento do

Tabela 1. Roteiro de conversa.

\begin{tabular}{cl}
$\begin{array}{c}\text { Temas } \\
\text { geradores }\end{array}$ & \multicolumn{1}{c}{ Questões norteadoras } \\
\hline Reabilitação & - O que você espera do processo de reabilitação? \\
\cline { 2 - 2 } Empoderamento & - Quais profissionais vieram te informar sobre a existência do processo de reabilitação? \\
\cline { 2 - 2 } Amputação & - Você está recebendo ajuda de alguém nessa fase de permanência no hospital? \\
\cline { 2 - 2 } Incertezas & - Você conhece alguém que sofreu uma amputação? \\
\cline { 2 - 2 } & - Alguém já te falou sobre reabilitação para pessoas amputadas? \\
\hline Orientação & - Você recebeu alguma orientação após a amputação? \\
\cline { 2 - 2 } & - Você acha importante receber orientação de algum profissional logo após a amputação? Por quê? \\
\hline
\end{tabular}

Fonte: Elaborada pelos autores. 


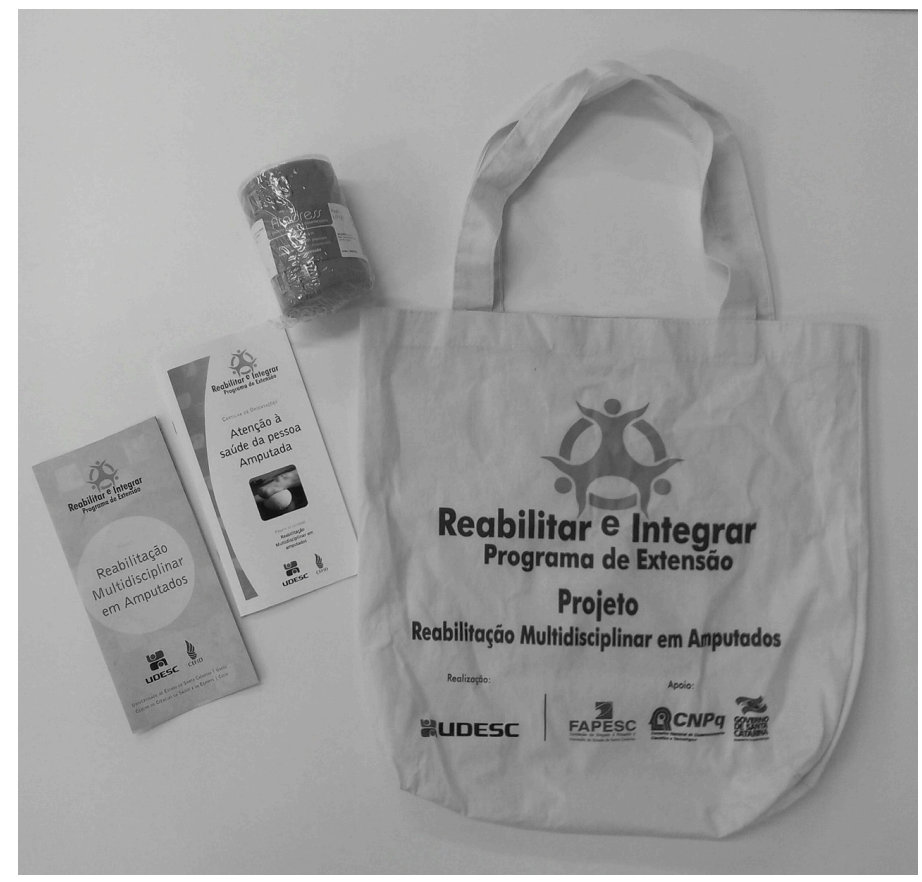

Figura 1. Kit confeccionado pelo projeto de extensão, UDESC. Fonte: Fotografada pelos autores.

coto após a alta hospitalar. A cartilha de orientaçôes foi elaborada a fim de orientar a pessoa no leito e após a alta hospitalar. Contém uma breve apresentaçáo sobre o projeto de extensáo, o local de funcionamento do projeto, um cartáo de agendamento da primeira consulta fisioterapêutica, informaçóes sobre dor e sensação fantasma, orientaçôes quanto a cuidados com o coto no pós-operatório imediato, cuidados posturais e enfaixamento do coto, informaçóes sobre o que é uma prótese e finaliza com instruçóes detalhadas de como conseguir uma prótese pelo Sistema Único de Saúde (SUS).

O folder sobre o projeto de extensão Reabilitação Multidisciplinar em Amputados traz informaçôes sobre o projeto, qual seu objetivo, local de funcionamento, atividades realizadas e a composição da equipe de trabalho.

Orientou-se nesta conversa o processo de aquisição da prótese pelo SUS, um dos processos que mais causam dúvidas e desinformação entre os profissionais de saúde e também às pessoas que sofreram amputação. $\mathrm{O}$ procedimento consiste em a pessoa amputada ou familiar contatar a Secretaria de Saúde do e esta identificar o local responsável em receber a prótese, levar ao local informado os seguintes documentos: solicitação para uso de prótese assinada pelo médico ou fisioterapeuta, cópia do Registro Geral (RG), Cadastro de Pessoa Física (CPF), comprovante de residência e cartão nacional do SUS.
A Secretaria municipal de Saúde do município de origem da pessoa recebe a solicitaçáo da prótese, verifica a documentação e abre o processo de solicitação, encaminhando a documentação para a respectiva Regional de Saúde. A Regional de Saúde (Secretaria Estadual de Saúde) recebe e analisa a documentação de cada processo de solicitação, encaminha o processo para o Centro Catarinense de Reabilitação (CCR) em Florianópolis - SC. O Centro Catarinense de Reabilitação (CCR) recebe o processo, agenda a primeira avaliação, avalia o caso, indica se a pessoa está apta à protetização, indica o tipo de prótese mais adequada de acordo com critérios técnicos, insere o usuário na lista de espera, chama para realizar medidas e entrega a prótese.

A faixa elástica compressiva para o enfaixamento do coto de amputaçáo foi incluída no kit, onde o manual orienta e conscientiza a pessoa sobre a importância do enfaixamento compressivo do coto de amputação após a alta hospitalar e ainda estimula a pessoa a procurar o projeto de extensão para aprender utilizar de forma adequada.

O enfaixamento compressivo do coto de amputação tem grande importância para o processo de reabilitação da pessoa amputada, pois evita o aumento do edema residual do coto, aumenta o metabolismo do coto, modelando e preparando-o para a protetização após a alta hospitalar (BRASIL, 2012b). 


\subsection{Procedimentos de análise de dados}

A análise da Conversa no Leito transcorreu da seguinte maneira: transcrição fidedigna do relato verbal e exploração dos dados e interpretação através da análise de conteúdo (BARDIN, 2009).

Os dados foram analisados integral e criteriosamente a fim de respeitar a fala da pessoa. A resposta da pessoa foi analisada por meio de um quadro analítico: Unidades de Significância, eixos das Unidades de Significância e eixos temáticos. Optou-se por essa proposta de análise porque os eixos possibilitam explorar o sentido da opiniáo coletiva de forma náo excludente, categorizadas.

Segundo Lefèvre e Lefevre (2005, p. 31), a análise de eixos é semanticamente mais rica, pois é mais plena de conteúdos significativos, fazendo emergir os variados detalhamentos individuais de uma mesma opiniáo coletiva diante do tema pesquisado. Primeiramente, o pesquisador criou unidades de significância em um processo de decodificação, por meio de eixos que demonstram ser pertinentes para responder aos objetivos desta pesquisa, ou seja, a fala do paciente foi analisada em consonância aos objetivos específicos, e assim foram classificadas nas Unidades de Significância.

\section{Resultados e Discussão}

A partir dos temas geradores Reabilitação; Empoderamento; Amputaçáo; Incertezas e Orientação, buscou-se refletir as falas da pessoa em relação à Reabilitação, que trouxe as seguintes questôes norteadoras:

Eu espero sair, me recuperar, se botar uma prótese, vai ser uma coisa realizada, eu poder andar.

Segundo a Diretriz de Atençáo à Saúde da Pessoa Amputada (BRASIL, 2012b), uma pessoa amputada unilateral com um bom condicionamento físico e com a cognição preservada já pode iniciar o treino de marcha e exercícios de descarga de peso no membro íntegro na fase pós-hospitalar imediata, para que ocorra uma habituação a sua nova condição física. $\mathrm{O}$ treino de marcha passa por etapas de progressão, sendo elas: barras paralelas, andador, muletas do tipo axilar ou canadense. Dentro desse treino, devem ser usados rampas, obstáculos, escadas e terrenos irregulares. O guideline inglês (BROOMHEAD et al., 2006) enfatiza que a reabilitaçâo se inicie no pós-operatório imediato se possível, com troca de posturas para evitar contraturas, transferências, ganho de mobilidade, além do uso de bandagens elásticas para a diminuição do edema do coto.

É bom colocar uma prótese, seria bom, ia me
ajudar, eu amo trabalhar.

Precisar voltar a trabalhar.

Volto a trabalhar eu já fico feliz.

Durante a Conversa no Leito, a pessoa relatou sua preocupação em voltar a trabalhar, realizar as atividades de que tanto gosta mas que foram, na concepção dela, limitadas pela amputaçáo. A diretriz brasileira nos mostra que a equipe multiprofissional é a responsável por informar os direitos das pessoas com deficiência e apresentar as possibilidades de retorno ao trabalho. Para guiar esses profissionais, em 2011 foi criado o Plano Nacional dos Direitos das Pessoas com Deficiência Viver sem Limites (BRASIL, 2012a), que tem como objetivo implementar novas iniciativas e intensificar açôes que beneficiam as pessoas com deficiência, e entre essas ações está a inserção no mercado de trabalho.

Em relação ao tema gerador Emponderamento, as seguintes falas nos foram remetidas:

Já vou começar, preparar a partir de hoje, me incentivar.

Segundo o guideline americado (UNITED..., 2007), a família tem um papel muito importante no incentivo à pessoa, pois ela é a responsável por incentivar um estilo de vida mais saudável, a realizaçáo de exercícios, alimentação adequada e, em alguns casos, a cessaçáo do tabagismo. Em contrapartida, a diretriz brasileira, relata que a troca de experiências e o contato com outras pessoas que sofreram uma amputação têm como objetivo o esclarecimento de dúvidas e a superação de dificuldades.

Em relação ao tema Amputação, as falas foram as seguintes:

\section{Eu sabia que ia perder.}

\section{Minha avó teve que amputar a perna.}

A etapa mais importante no processo de reabilitação é o momento da notícia, é necessária uma equipe preparada para dar a notícia, informar a pessoa e seus familiares sobre a sua nova situação. Nos casos de cirurgias eletivas, o apoio psicológico prévio à pessoa auxilia-a a lidar com o processo operatório, a aceitar o processo de reabilitação e de protetização (BRASIL, 2012b). Quando o indivíduo é submetido a uma amputação de urgência, por exemplo, após um acidente automobilístico, não irá ocorrer o 
momento da notícia à pessoa, devido à urgência em realizar o procedimento cirúrgico por causa do estado geral da pessoa. Intervençôes educativas pré-operatórias devem ser fornecidas à pessoa antes da amputação, processo que irá diminuir seus medos, ansiedades e angústias, melhorando sua recuperação no pós-operatório (UNITED..., 2007).

Não posso fazer nada, chorar, não podia me desesperar.

Fico triste às vezes, bola pra frente.

Segundo Sebastiani e Maia (2005), o processo cirúrgico acarreta mudanças na vida e no estado emocional da pessoa que sofreu uma amputaçáo. A pessoa sente-se fragilizada, emocionalmente instável, tem dúvidas de como será o pós-operatório, de como ficará seu corpo e medo de ser tornar incapaz. O guideline americano (UNITED..., 2007) ressalta que o funcionamento psicossocial deve ser avaliado em cada fase do processo de reabilitação, deve centrar-se sobre os sintomas atuais e passados, principalmente depressão, ansiedade e sintomas de estresse pós-traumático.

No momento da conversa, a pessoa relatou poucas incertezas quanto ao processo de reabilitação.

\section{Não tenho muitas dúvidas.}

\section{Dúvidas sobre o amanhã, sobre hoje.}

Um estudo também de caráter qualitativo realizado por Pedlow et al. (2014) com pessoas submetidas a uma amputação de membros inferiores secundária de uma doença vascular teve como objetivo explorar as perspectivas das pessoas que foram submetidas à cirurgia de amputação. Em relação às informações fornecidas durante o processo de internaçáo pelos profissionais de saúde, as pessoas relataram que não havia informaçóes suficientes em uma variedade de tópicos, incluindo o processo de recuperação, as expectativas para a reabilitação e a prótese. As pessoas tinham diferentes perspectivas sobre a pertinência das informaçôes, tipo de cirurgia e a quantidade de informaçôes fornecidas.

Percebe-se que se faz necessário uma abordagem individualizada para o fornecimento de informaçóes a pessoas submetidas a uma amputação de membros inferiores, e que a família tem papel importante nesse processo.

A presença da família no ambiente hospitalar junto à pessoa é de extrema importância, pois auxilia nas tomadas de decisóes e retiradas de dúvidas (LUSTOSA, 2007). Uma equipe multiprofissional é a mais capacitada para sanar as incertezas da pessoa hospitalizada, em especial o psicólogo hospitalar, que possui um papel importante na retirada de dúvidas da pessoa e auxilia no estabelecimento do vínculo entre equipe/família/pessoa (MOREIRA; MARTINS; CASTRO, 2012).

Durante o diálogo, a pessoa relatou sobre as orientaçóes que recebeu no ambiente hospitalar.

\section{O rapaz do seguro veio aqui, rapaz do seguro DPVAT.}

\section{De encaminhação [encaminhamento] de falar em tal lugar não falaram.}

\section{Os médicos não vieram.}

Geertzen et al. (2015a) sugerem que as orientaçóes devem ser adaptadas às necessidades de cada pessoa, e a forma como essas orientaçóes serão fornecidas também terá de ser adaptada a cada pessoa.

É importante destacar à pessoa os objetivos do tratamento fisioterapêutico, informar sobre a equipe que irá auxiliá-la, e o envolvimento dos familiares nesse processo é de extrema importância. O fisioterapeuta é um dos responsáveis pelas orientaçóes à pessoa sobre todo o processo de reabilitaçáo, desde os locais onde são fornecidos programa de reabilitação, informaçôes sobre o processo de protetização, seus benefícios e demonstração do uso correto da prótese, até o suporte para a manutenção desta e informar sobre a limitação funcional de cada pessoa a partir do seu nível de amputação (BROOMHEAD et al., 2006).

\section{Conclusão}

A ação educativa individualizada no ambiente hospitalar chamada Conversa no Leito contribuiu a partir dos cinco temas geradores: Reabilitação; Empoderamento; Amputação; Incertezas e Orientação para a promoção da saúde da pessoa amputada. Mostrou-se eficiente principalmente no empoderamento da pessoa para o início precoce da reabilitação. Salienta-se atenção para capacitação da equipe multidisciplinar hospitalar envolvida quanto a orientaçóes sobre protetização, retorno ao trabalho e esclarecimento de dúvidas gerais da pessoa, assim como atençấo aos seus anseios e suporte familiar.

\section{Referências}

BARDIN, L. Análise de conteúdo. Lisboa: Ediçôes, 2009.

BRASIL. Ministério da Saúde. Saúde em Primeiro Plano. Brasília: Ministério da Saúde, 2005. Disponível em: <http://portal.saude.gov.br/portal/arquivos/pdf/saude_ brasil_dezembro.pdf>. Acesso em: 1 jun 2015. 
BRASIL. Secretaria Especial dos Direitos da Pessoa com Deficiência. Plano Nacional dos Direitos da Pessoa com Deficiência: Viver Sem Limite. Brasília: Secretaria Especial dos Direitos da Pessoa com Deficiência, 2012a. Disponível em: <http://www.brasil.gov.br/viversemlimite/plano-nacional-dos-direitos-da-pessoa-com-deficiencia>. Acesso em: 22 maio 2016.

BRASIL. Ministério da Saúde. Diretrizes de atenção à pessoa amputada. Brasília: Ministério da Saúde, 2012b.

BRASIL. Ministério da Saúde. Departamento de Informática do SUS - Datasus. Morbidade Hospitalar do SUS por Causas Externas - por local de internação - Brasil. Brasília: Ministério da Saúde, 2015.

BROOMHEAD, P. et al. Clinical guidelines for the pre and post operative physiotherapy management of adults with lower limb amputation. London: BACPAR, 2006. Disponível em: <http://bacpar.csp.org.uk/publications/ clinical-guidelines-pre-post-operative-physiotherapy-management-adults-lower-li>. Acesso em: 25 jan 2017.

CARVALHO, F. S. et al. Prevalência de amputação em membros inferiores de causa vascular: análise de prontuários. Arquivos de Ciências da Saúde da UNIPAR, Umuarama, v. 9, n. 1, p. 23-30, 2005.

CHINI, G. C. O.; BOEMER, M. R. A amputação na percepção de quem a vivencia: um estudo sob a ótica fenomenológica. Revista Latino-Americana de Enfermagem, Ribeirão Preto, v. 15, n. 2, p. 330-336, 2007.

GASKELL, G.; BAUER, M. W. Pesquisa qualitativa com texto, imagem e som: um manual prático. Petrópolis: Vozes, 2002.

GEERTZEN, J. et al. Dutch evidence-based guidelines for amputation and prosthetics of the lower extremity: Amputation surgery and postoperative management. Part 1. Prosthetics and Orthotics International, London, v. 39, n. 5, p. 351-360, 2015a.

GEERTZEN, J. et al. Dutch evidence-based guidelines for amputation and prosthetics of the lower extremity: Rehabilitation process and prosthetics. Part 2. Prosthetics and Orthotics International, London, v. 39, n. 5, p. 361371, 2015b.

LEFÈVRE, F.; LEFEVRE, A. M. C. Depoimentos e discursos: uma proposta de análise em pesquisa social. Brasília: Líber Livro, 2005.

LUSTOSA, M. A. A família do paciente internado. Revista da Sociedade Brasileira de Psicologia Hospitalar, Rio de Janeiro, v. 10, n. 1, p. 3-8, 2007.

MOREIRA, E. K. C. B.; MARTINS, T. M.; CASTRO, M. M. Representação social da Psicologia Hospitalar para familiares de pacientes hospitalizados em Unidade de Terapia Intensiva. Revista da Sociedade Brasileira de Psicologia Hospitalar, Rio de Janeiro, v. 15, n. 1, p. $134-$ 167, 2012.

PEDLOW, H. et al. Patient perspectives on information needs for amputation secondary to vascular surgery: What, when, why, and how much? Journal of Vascular Nursing, Norwood, v. 32, n. 3, p. 88-98, 2014.

SEBASTIANI, R. W.; MAIA, E. M. C. Contribuições da psicologia da saúde-hospitalar na atenção ao paciente cirúrgico. Acta Cirúrgica Brasileira, Sáo Paulo, v. 20, p. 50-55, 2005.

SILVA, M. A. M. et al. Promoção da saúde em ambientes hospitalares. Revista Brasileira de Enfermagem, Brasília, v. 64, n. 3, p. 596-599, 2011.

SOUZA, M. C. M. O desafio do conhecimento: pesquisa qualitativa em saúde. São Paulo: Hucitec, 2013.

STAKE, R. E. Investigación con estudio de casos. Madrid: Morata, 2005.

SZYMANSKI, H.; ALMEIDA, L. R.; PRANDINI, R. C. A. R. A entrevista na pesquisa em educação: a prática reflexiva. Brasília: Liber Livro, 2010.

UNITED STATES DEPARTMENT OF VETERAN AFFAIRS. VA/DoD clinical practice guideline for rehabilitation of lower limb amputation. Washington: Department of Veteran Affairs, 2007.

\section{Contribuição dos Autores}

Ruy Branco e Kadine Bender: participaram da coleta, análise de dados e da elaboração do artigo. Soraia da Luz: participou da orientação do trabalho e revisão do artigo. Todos os autores aprovaram a versão final do texto.

\section{Fonte de Financiamento}

Apoio financeiro da Fundação de Amparo à Pesquisa do Estado de Santa Catarina (FAPESC), Brasil, processo no 3656/2013 e da Chamada Universal (CNPq), Brasil, processo no 14/2013. 